\title{
Levantamento de seio maxilar utilizando osso liofilizado associado a instalação imediata de implante do tipo cone morse: relato de caso
}

\author{
Survey of maxillary sinus using lyophilized bone associated with \\ immediate implant placement taper morse: a case report
}

\author{
Assis Filipe Medeiros Albuquerque* \\ Ivson Macêdo Lopes Cardoso ${ }^{* *}$ \\ José Sandro Pereira da Silva*** \\ Adriano Rocha Germano ${ }^{* * * *}$ \\ Wagner Ranier Maciel Dantas ${ }^{* * * *}$ \\ André Luiz Marinho Falcão Gondim ${ }^{* * * * *}$
}

\begin{abstract}
Resumo
Objetivo: apresentar um caso clínico de levantamento de seio maxilar, utilizando biomaterial e instalação de implante imediato e discutir alguns fatores importantes para o sucesso desse tipo de tratamento. Relato de caso: Paciente do sexo masculino, 65 anos de idade, procurou o Serviço de prótese sob implante e implantodontia com o objetivo de reabilitar área edêntula na região do dente 15 com implante. No exame de imagem foi observado pouca altura óssea remanescente para realização do implante na região. Diante das características do caso, foi proposta para o paciente a realização do levantamento de seio com a utilização de enxerto ósseo particulado (biomaterial) simultâneo à colocação de implante na área, visando uma reabilitação mais rápida do paciente. Atualmente, o paciente encontra-se com 12 meses de pós-operatório, com reabilitação final concluída, bons resultados estético e funcional foram conseguidos. Os exames de imagem apresentam total incorporação do implante ao leito receptor e ao enxerto realizado após 1 ano de acompa-
\end{abstract}

nhamento. Considerações Finais: Pode-se concluir que a instalação de implante imediato após a realização do levantamento do seio maxilar, é um procedimento viável, quando bem indicado, e que proporciona ao paciente uma diminuição do tempo de tratamento.

Palavras-chave: Levantamento de seio maxilar. Enxerto ósseo. Implante dentário.

\section{Introdução}

A utilização de implantes dentários tem sido a escolha na maioria dos casos para reabilitação dos espaços edêntulos. No entanto, nas regiões que houve perdas dentárias, ocorre um grau de reabsorção óssea, podendo variar de moderada à severa, o que muitas vezes impossibiliita a instalação direta dos implantes. Dessa forma, deve-se lançar mão de re-

\footnotetext{
http://dx.doi.org/10.5335/rfo.v19i1.3621
}

DDS, Mestrando em Odontologia - Área de CTBMF pela Universidade Federal do Ceará, Residência em CTBMF pela Universidade Federal do Rio Grande do Norte - UFRN, Departamento de Odontologia, Área de CTBMF, Natal, RN, Brasil.

DDS, Especialista em Reabilitação Oral - ABO/RN, Departamento de Saúde do Corpo de Bombeiros Militar do Rio Grande do Norte, Natal, RN, Brasil.

DDS, Ms, PhD, Doutor em ciências pela USP/SP. Professor adjunto II da área de Cirurgia e Traumatologia Buco-Maxilo-Facial e coordenador da setor de Implantodontia do Departamento de Odontologia da Universidade Federal do Rio Grande do Norte, Natal, RN, Brasil.

DDS, Ms, PhD, Doutor em CTBMF pela UNICAMP. Professor associado I da área de Cirurgia e Traumatologia Buco-Maxilo-Facial da Universidade Federal do Rio Grande do Norte e Coordenador do Serviço de Residência em CTBMF do Departamento de Odontologia e Hospital Universitário Onofre Lopes (DOD HUOL/UFRN), Natal, RN, Brasil.

***** DDS, Ms, PhD, Pós-Doutorado em Patologia Bucal pela FOUSP. Professor adjunto da área de CTBMF do Departamento de Odontologia da Universidade Federal do Rio Grande do Norte, Natal, RN, Brasil.

DDS, Ms, PhD, Doutorado em CTBMF pela Pontifícia Universidade Católica do Rio Grande do Sul. Professor Adjunto II da área de CTBMF do Departamento de Odontologia da Universidade Federal do Rio Grande do Norte, Natal, RN, Brasil. 
construções ósseas, seja com material autógeno, biomateriais ou associação de ambos ${ }^{1}$.

A perda de dentes na região posterior da maxila e o avanço da idade do paciente, apresentam como consequências, além da reabsorção óssea do osso alveolar, a pneumatização dos seios maxilares, o que muitas vezes inviabiliza a instalação dos implantes. Além disso, a região posterior da maxila apresenta uma qualidade óssea deficiente, com cortical delgada e trabeculado extremamente poroso ${ }^{2}$. A reabilitação bucal com o uso de implantes dentários, nessa região, é frequentemente dificultada por essa deficiência quantitativa e qualitativa da estrutura óssea remanescente. Considerada como dificuldade adicional, no interior das cavidades dos seios maxilares, há restrições que podem complicar o posicionamento ideal dos implantes. Essas incluem, inclinações do assoalho da cavidade e a presença de septos ósseos na região ${ }^{3}$.

Para minimizar e possibilitar a reabilitação na região posterior de maxila, podemos usar duas técnicas de levantamento do assoalho do seio maxilar: a abordagem através da confecção de uma janela óssea na parede lateral e a abordagem via alveo$\operatorname{lar}^{3}$. A escolha da técnica a ser utilizada, será baseada na estrutura óssea residual remanescente e no quanto de levantamento do assoalho será necessário. O espaço criado abaixo da membrana é então enxertado e/ou implantado com o uso de osso autógeno, osso halógeno, biomateriais ou a combinação desses. Os implantes dentários podem ser inseridos durante o processo de enxertia, imediato, ou após um período de cicatrização primária que varia de quatro a 12 meses $^{4}$.

$\mathrm{Na}$ utilização do enxerto autógeno, apesar de ser considerado o padrão ouro por ter as qualidades de osteogênese, osteoindução e osteocondução, nos deparamos com algumas limitações como a quantidade requerida, principalmente por via intra bucal além de apresentar uma maior morbidade relacionada à necessidade de um segundo sítio doador e um maior desconforto ao paciente no pós-operatório. Considerando esses fatores, os biomateriais, estão sendo amplamente utilizados, por apresentarem propriedades osteoindutoras e osteocondutoras e possuírem uma quantidade e tipo diversos, podem ser utilizados em qualquer tipo de reconstrução, visando a instalação de implantes, além de não proporcionar maior morbidade ao paciente e ter atualmente uma previsibilidade de resultados a longo prazo bastante satisfatório ${ }^{1,5}$.

Nas reabilitações na região do seio maxilar, no qual o remanescente ósseo for maior ou igual a 4 $\mathrm{mm}$ de altura, na qual se consiga obter uma adequada estabilidade primária, fator imprescindível para o sucesso da osseointegração, o implante poderá ser realizado de maneira simultânea à colocação do enxerto ósseo, durante o levantamento da membrana de Schneide. Já, no caso de um remanescente menor que $4 \mathrm{~mm}$ de altura, é indicada a realização inicialmente do enxerto ósseo, e, em seguida, após 6-10 meses dependendo do material utilizado, realizar a instalação do implante ${ }^{4}$.

O objetivo desse artigo é apresentar um caso clínico de uma cirurgia de levantamento do seio maxilar, utilizando biomaterial (osso bovino liofilizado), com instalação imediata de implante e reabilitação final.

\section{Relato de caso}

Paciente do sexo masculino, 65 anos de idade, procurou o Serviço de prótese sob implante e implantodontia da Faculdade de Odontologia da UFRN com o objetivo de reabilitar área edêntula na região do dente 15 com implante. Durante o exame clínico intra-bucal, foi observado uma distância mésio distal de $9 \mathrm{~mm}$, favorecendo a correta reabilitação protética, porém apresentava uma pneumatização do seio maxilar na região. Na tomografia computadorizada se constatou um seio maxilar saudável, com remanescente ósseo satisfatório no sentido vestibulo-palatino de $6,50 \mathrm{~mm}$, e uma altura óssea remanescente de $5,60 \mathrm{~mm}$, possibilitando assim a realização do levantamento de seio maxilar e implante imediado associado ao enxerto ósseo particulado (Fig. 1).

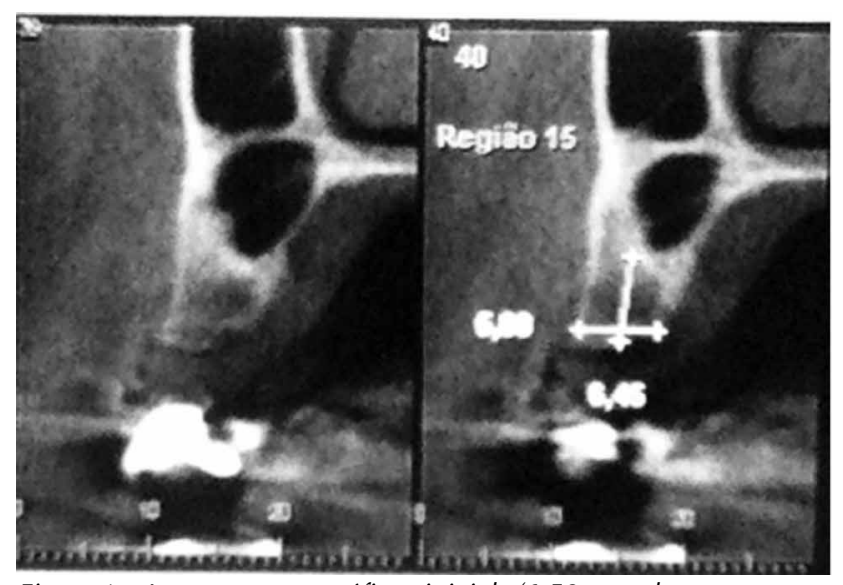

Figura 1 - Imagem tomográfica inicial $(6,50 \mathrm{~mm}$ de espessura e $5,60 \mathrm{~mm}$ de altura)

A técnica cirúrgica consistiu de uma anestesia infiltrativa na região com mepivacaina $2 \%$ e epinefrina 1:100.000, incisão sobre a crista extendendo-se com uma incisão intrasulcular na região do dente 16 e uma incisão relaxante na mesial do dente 14. Após o descolamento mucoperiosteal de espessura total, foi realizado o desgaste da parede anterior do seio maxilar com broca esférica diamantada, deixando a membrana do seio exposta (Fig. 2). Foi realizado o descolamento cuidadoso da membrana do seio com curetas específicas sem corte, mantendo a membrana na região superior após todo o descolamento, para que fosse realizada a colocação do enxerto, sem haver a perfuração dessa (Fig. 3). 


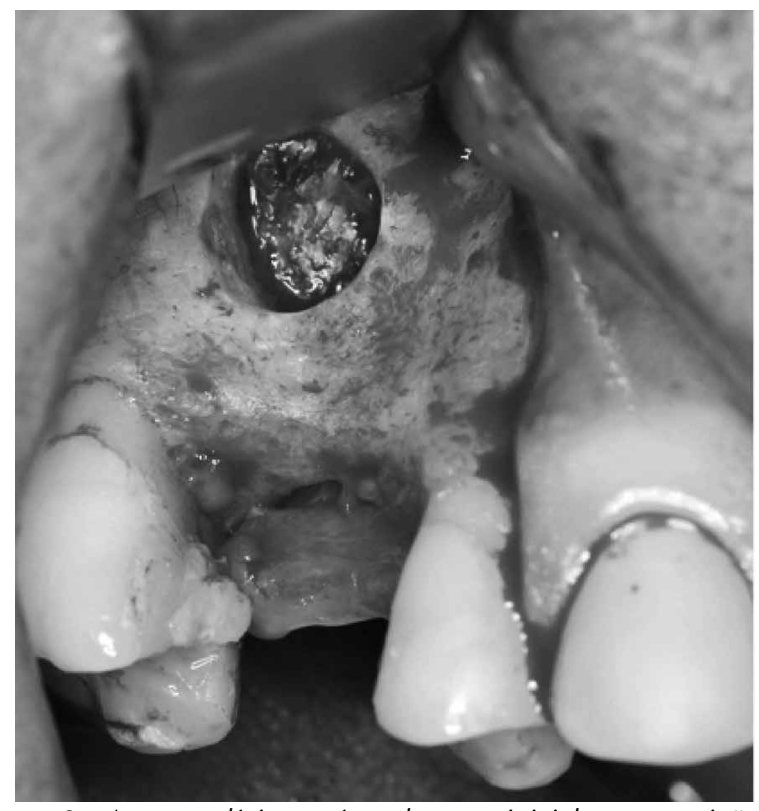

Figura 2 - Aspecto clínico após o desgaste inicial e a exposição da membrana sinusal

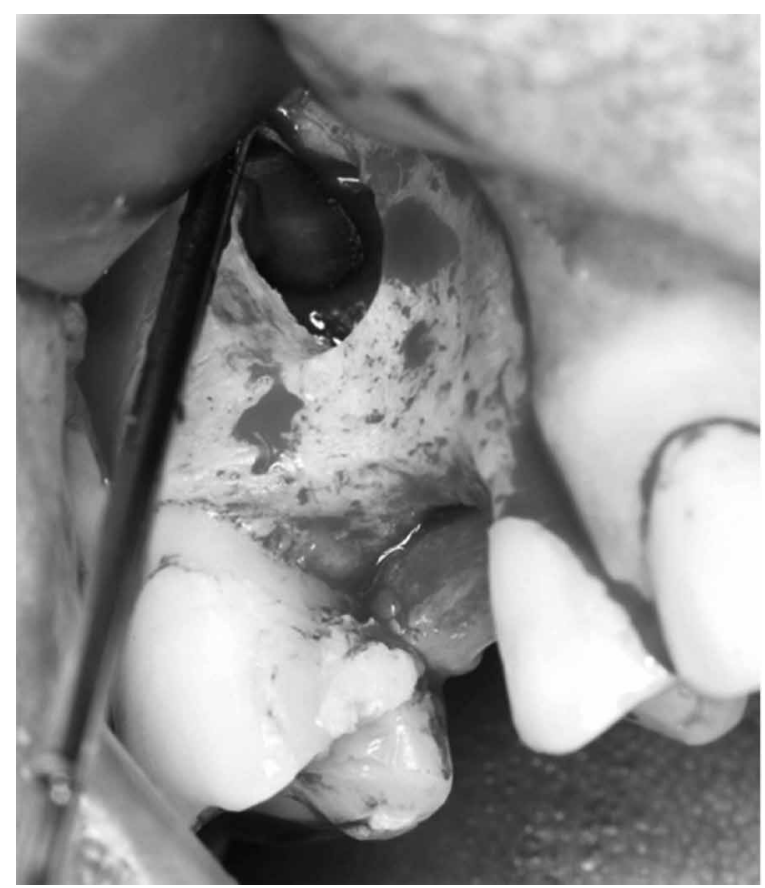

Figura 3 - Aspecto clínico do descolamento cuidadoso da membrana sinusal

Selecionado o implante do tipo Drive Cone morse (Ref. 109.627, Neodent ${ }^{\circledR}$ - Curitiba, PR, Brasil), $4,3 \mathrm{~mm}$ de diâmetro e $11,5 \mathrm{~mm}$ de comprimento, realizamos a instrumentação para receber o implante. Após a finalização do preparo para tal, foi realizado o preenchimento da porção distal e do palatino do assoalho do seio maxilar, com o biomaterial Geistlich Bio-Oss ${ }^{\circledR}$ (Ref. 30643.3, Geistlich, Switzerland, Suiça), seguido da instalação do implante (Fig. 4), que obteve um torque de $60 \mathrm{~N} . \mathrm{cm}$, aferido com a catraca torquímetro (Ref. 104.050, Neodent ${ }^{\circledR}$ - Curitiba, PR, Brasil). E então todo o restante da cavidade foi preenchido com o Bio-Oss ${ }^{\circledR}$ (Fig. 5), e, sobre a janela realizada, e o biomaterial exposto, foi adicionado uma membrana de colageno absorvível Geistlich
Bio-Guide ${ }^{\circledR}$ (Ref. 30802.6, Geistlich, Switzerland, Suiça) (Fig. 6). O retalho foi então reposicionado e suturado com fio de seda 3.0 (Ref. SO34CT17, Technofil, Goiânia, Goiás, Brasil) (Fig. 7).

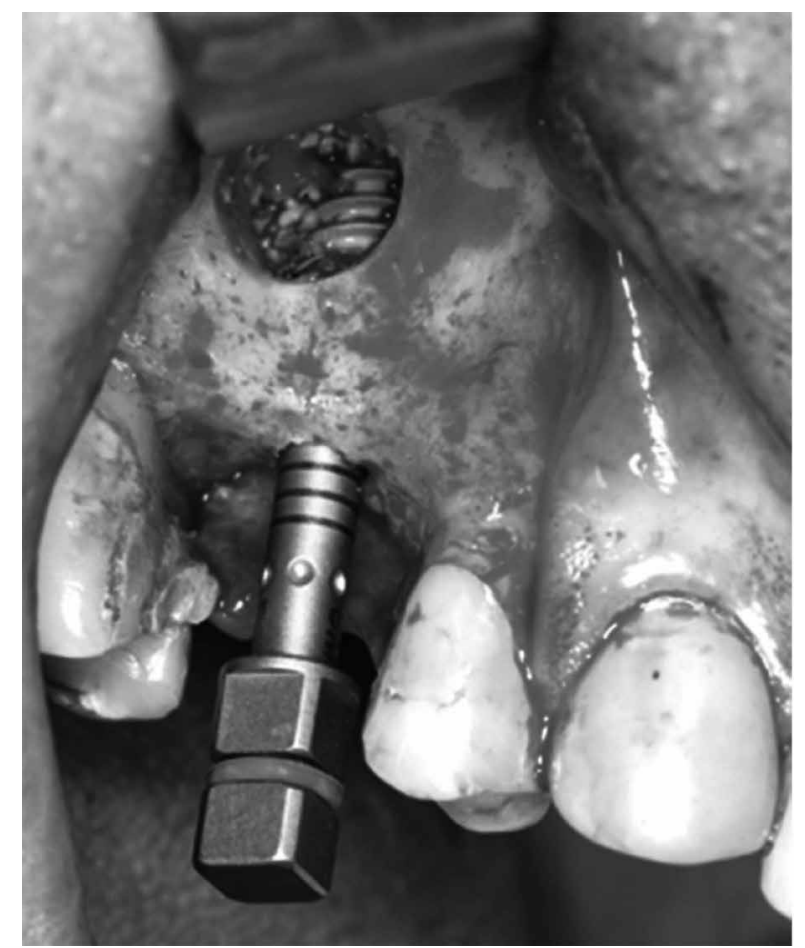

Figura 4 - Preenchimento da porção distal e palatina com Bio-Oss $®$ e inserção do implante

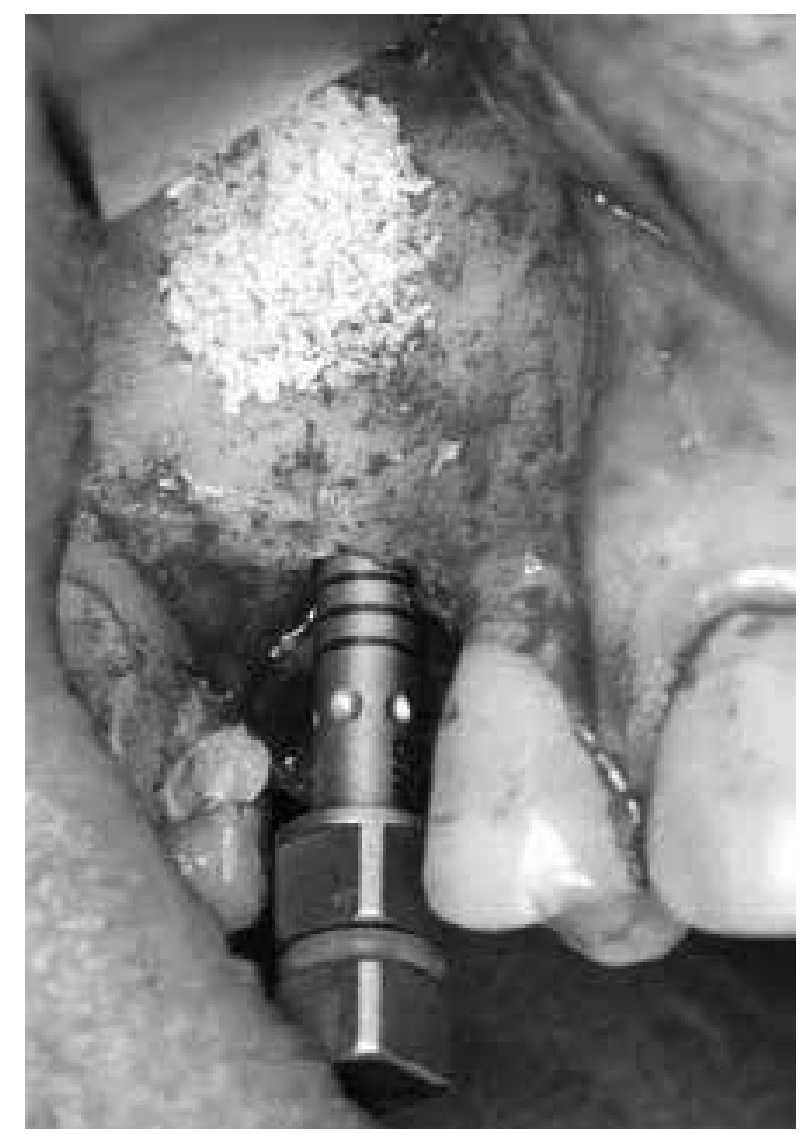

Figura 5 - Preenchimento de toda a cavidade com Bio-Oss® 


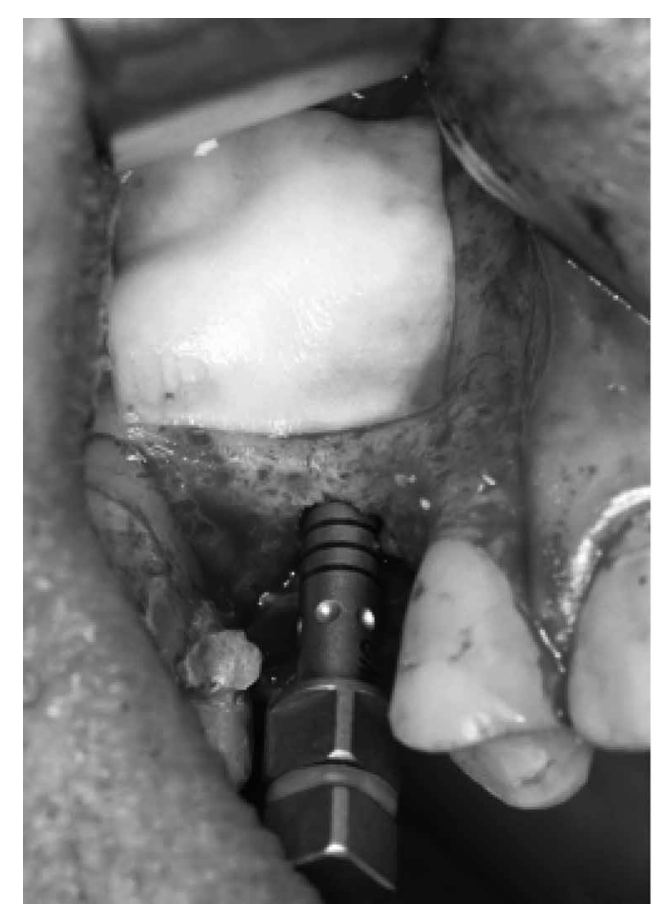

Figura 6 - Colocação da membrana reabsorvível (Bio-Guide®)

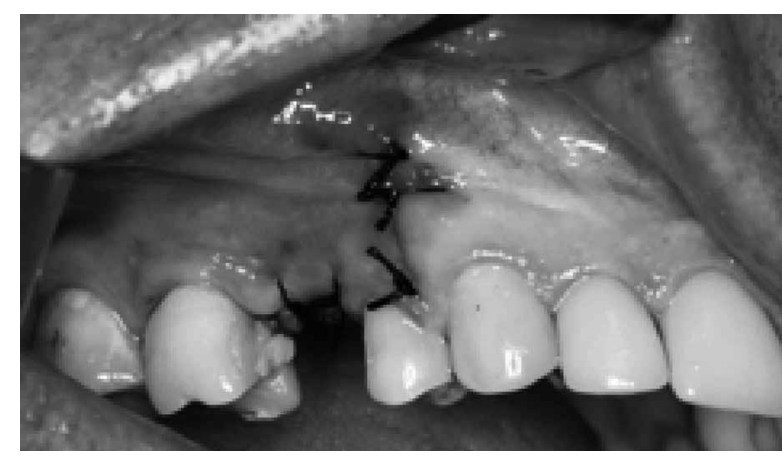

Figura 7 - Aspecto clínico após a realização da sutura

Após 7 meses da realização do enxerto e da colocação do implante foi realizado a instalação da protese. E passados 12 meses de pós-operatório, observa-se boa estabilidade do implante em condições favoráveis e em função (Fig. 8). Os exames radiográfico e tomográfico pós-operatórios de 12 meses, mostraram uma adequada formação óssea ao redor de todo o implante (Fig. 9). Possibilitou-se assim a adequada reabilitação da região, com contorno gengival e perfil de emergência adequados, resultando em um bom resultado estético funcional.
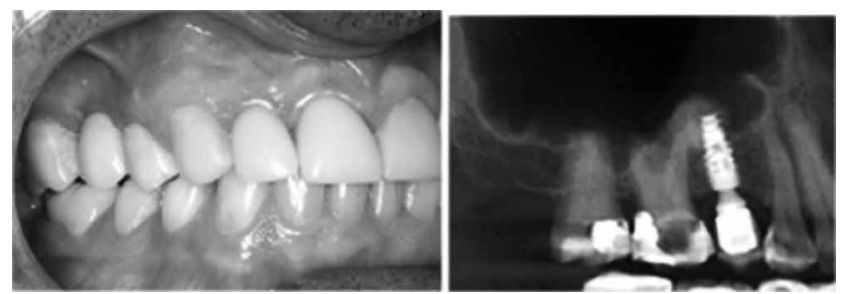

Figura 8 - Reabilitação finalizada com a prótese, com 12 meses em função

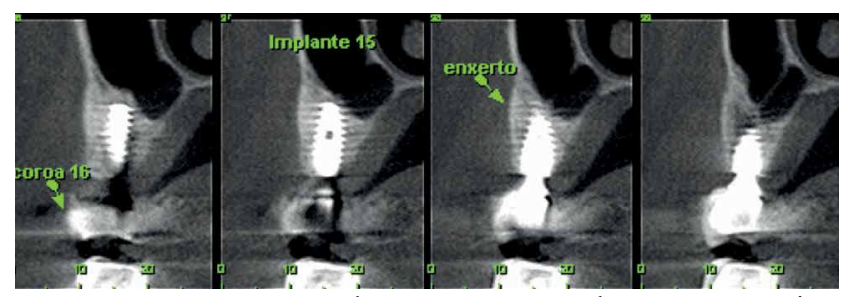

Figura 9 - Imagem tomográfica após 1 ano em função, mostrando a total incorporação do enxerto e implante

\section{Discussão}

Os vários biomateriais disponíveis indicados para o levantamento do seio maxilar apresentam diferentes comportamentos biológicos de acordo com origem, forma, tamanho, porosidade e taxa de degradação. Essas diferenças atuam diretamente sobre a taxa e o tempo de formação do osso. O Bio-Oss ${ }^{\circledR}$ é um xenoenxerto bovino desproteinado, de reabsorção lenta, química e fisicamente idêntico ao osso humano, em forma de grânulos corticais, apresentando $75 \%$ a $80 \%$ de porosidade e uma vasta rede de interligação do sistema de macro e micro-poros, que facilita a angiogênese e a migração dos osteoblastos. Algumas investigações reafirmaram que a breve presença do Bio-Oss ${ }^{\circledR}$ incorporado ao osso esponjoso cria uma densa rede através do reforço da massa de tecido ósseo e melhora a sua capacidade de suportar as forças da carga transmitida por implantes dentários ${ }^{6}$.

Quando se avalia a utilização dos biomateriais como o enxerto ósseo, temos alguns estudos na literatura bastante importantes, como o de Mangano et al. ${ }^{7}$ (2007), dividiu seu trabalho em dois grupos: grupo 1 que recebeu enxerto ósseo derivado-bovino (Bio-oss ${ }^{\circledR}$; Geistlich, Wohlhusen, Switzerland) e grupo 2 que recebeu hidroxiapatita (HA) (Fingranule ${ }^{\circledR}$; Finceramica, Faenza, Ravena, Itália). No grupo 1 a maioria das partículas estava rodeada por neoformação óssea. Esse osso apresentou-se maduro e compacto com uma estrutura bem organizada de esteócitos, sem espaços na interface osso-partícula, e o osso sempre em íntimo contato com as partículas. Não foram encontradas células inflamatórias ou células gigantes multinucleadas. Canais de $h a-$ vers estavam presentes para serem colonizados por capilares e células. Já no grupo 2 também houve formação de osso novo com largas lacunas de osteócitos rodeadas por partículas de HA, que pareciam estar sendo reabsorvidas em reposta ao osso novo. Várias células multinucleadas foram encontradas nas superfícies das partículas da HA. Osso lamelar e sistema de harves estavam presentes. Não foram encontradas células inflamatórias. Osteoblastos foram observados perto da partículas de HA secretando matriz osteóide. Não houve espaços na interface osso-partícula, o que mostra que a nossa opção pela utilização do Bio-Oss ${ }^{\circledR}$ vem se demostrando bem 
aceita na literatura, com bons resultados nas reconstruções ósseas ${ }^{7}$.

Outros trabalhos mostram também bons resultados quando da realização do implante imediato ao levantamento do seio maxilar e a utilização de biomaterial: Scarano et al. ${ }^{8}$ inseriram 362 implantes em 94 pacientes e tiveram apenas seis implantes perdidos; taxa de sucesso de 98,35\%; Peleg et al. ${ }^{9}$ em um acompanhamento a longo prazo, por nove anos, foram colocados 2.132 implantes desses, 44 falharam, representando uma taxa de sucesso de 97,9\%. Mangano et al. ${ }^{7}$, colocaram 100 implantes em dois tipos de biomateriais e tiveram quatro falhas, o que representa uma taxa de sucesso de $96 \%$.

Relacionado à técnica utilizada no caso, optamos por realizar a técnica em um estágio, realizando-se a inserção do implante no mesmo momento do enxerto ósseo, diminuindo assim, o tempo de tratamento até a reabilitação final para o paciente, haja visto que apresentava um leito adequado, com remanescente ósseo suficiente e saúde do seio maxilar, o que proporcionou a realização dessa técnica. Apresentando um bom resultado até o presente momento, corroborando com o estudo de Hürzeler et al. ${ }^{10}$, que realizaram 340 implantes; desses, 235 foram imediatos e 105 foram tardios. Todos os implantes osseointegraram e nenhum implante falhou antes da reabilitação protética. Dos 340 implantes colocados, 33 falharam ao longo do período de proservação; dois implantes exibiram mobilidade oito implantes foram considerados falhas por demonstrar áreas radiolucidas ao redor; 19 implantes apresentaram perda óssea maior que $0,2 \mathrm{~mm}$ por ano e quatro implantes falharam pela combinação de duas ou mais razões, como mobilidade, radiolucidez, perda óssea vertical. Esse estudo também confrontou as falhas de implantes com as alturas ósseas residuais: em altura óssea residual menor que $4 \mathrm{~mm}$ perderam-se três implantes; menor que $6 \mathrm{~mm}$ perderam-se oito implantes; menor que $8 \mathrm{~mm}$ houve 13 falhas e em maiores de $8 \mathrm{~mm}$ houve oito falhas ${ }^{10}$.

A perfuração da membrana sinusal é uma complicação transoperatória frequente e que contribui com as taxas de insucesso no resultado final das cirurgias de levantamento de seio maxilar e dos implantes. E a ocorrência dessas perfurações pode estar relacionada a fatores locais, cúpulas radiculares, presença de septos ósseos, além de patologias sinusais prévias como o tabagismo. A perfuração da membrana não é uma contra indicação absoluta para prosseguir com o procedimento, pois perfurações menores que $10 \mathrm{~mm}$ de extensão, podem ser tratadas e o reparo dessas perfurações podem envolver o uso de membrana reabsorvível, membrana de colágeno e sutura. No nosso caso, não houve perfuração da membrana sinusal, fato este que promove melhor sobrevida ao implante ${ }^{11,12}$.

\section{Conclusão}

O caso mostra a possibilidade viável da realização do levantamento de seio maxilar associado a enxerto ósseo com biomaterial (Bio-Oss ${ }^{\circledR}$ ) e a instalação simultânea de implante na região. Alguns fatores são essenciais para o sucesso do caso, dentre eles podemos citar a quantidade de remanescente ósseo, a estabilidade primária do implante no momento da instalação, a manutenção da integridade da membrana sinusal, a saúde do seio maxilar prévio, a cirurgia e o material utilizado.

\section{Abstract}

Objective: to present a clinical case of maxillary sinus lifting using biomaterial and immediate implant placement, and to discuss some relevant factors to a successful treatment. Case Report: Male patient, 65 years old, came to the prosthesis over implant and Implantology Service with the goal of rehabilitating edentulous area in tooth 15 region with implant. Imaging examination revealed little residual bone height to place the implant in the region. Given the characteristics of the case, it was proposed to the patient the performance of maxillary sinus lifting using lyophilized bone graft (biomaterial) along with immediate implant placement, aiming at a faster rehabilitation of the patient. Currently the patient is at 12-month post-operative period, he concluded final rehabilitation, and presents satisfactory esthetic and functional results. Imaging exams show complete incorporation of the implant to the recipient site and to the bone graft after 1 year of follow-up. Final Considerations: It may be concluded that immediate implant placement after maxillary sinus lifting is a viable procedure when properly indicated, providing reduced treatment time to the patient.

Keywords: Maxillary sinus lifting. Bone graft. Dental implants.

\section{Referências}

1. Raja SV. Management of the posterior maxilla with sinus lift: review of techniques. J Oral Maxillofac Surg 2009; 67(8):1730-4.

2. Small SA, Zinner ID, Panno FV, Shapiro HJ, Stein JI. Augmenting the maxillary sinus for implants: report of 27 patients. Int J Oral Maxillofac Implants 1993; $8(5): 523-8$

3. Chen L, Cha J. An 8-year retrospective study: 1.100 patients receiving 1.557 implants using the minimally invasive hydraulic sinus condensing technique. J Periodontol 2005; 76(3):482-91.

4. Chen TW, Chang HS, Leung KW, Lai YL, Kao SY. Implant placement immediately after the lateral approach of the trap door window procedure to create a maxillary sinus lift without bone grafting: a 2-year retrospective evaluation of 47 implants in 33 patients. J Oral Maxillofac Surg 2007; 65(11):2324-8. 
5. Voss P, Sauerbier S, Wiedmann-Al-Ahmad M, Zizelmann C, Stricker A, Schmelzeisen R, Gutwald R. Bone regeneration in sinus lifts: comparing tissue-engineered bone and iliac bone. Br J Oral Maxillofac Surg 2010; 48(2):121-6.

6. De Souza Nunes LS, De Oliveira RV, Holgado LA, Nary Filho H, Ribeiro DA, Matsumoto MA. Use of bovine hydroxyapatite with or without biomembrane in sinus lift in rabbits: histopathologic analysis and immune expression of core binding factor 1 and vascular endothelium growth factor. J Oral Maxillofac Surg 2011; 69(4):1064-9.

7. Mangano C, Scarano A, Perrotti V, Iezzi G, Piattelli A. Maxillary sinus augmentation with a porous synthetic hydroxyapatite and bovine-derived hydroxyapatite: a comparative clinical and histologic study. Int J Oral Maxillofac Implants 2007; 22(6):980-6.

8. Scarano A, Degidi M, Iezzi G, Pecora G, Piattelli M, Orsini G. Maxillary sinus augmentation with different biomaterials: a comparative histologic and histomorphometric studijin man. Implant Dentistry 2006; 15(2):197-207.

9. Peleg M, Garg AK, Mazor Z. Predictability of simultaneous implant placement in the severely atrophic posterior maxilla: a 9-year longitudinal experience study of 2.132 implants placed into 731 human sinus grafts. Int J Oral Maxillofac Implants 2006; 21(1):94102.

10. Hürzeler MB, Kirsch A, Ackermann KL, Quiñones CR. Recontruction of the severely redorbed maxilla with dental implants in the augumented maxillary sinus: a 5-year clinical investigation. Int J Oral Maxillofac Implants 1996; 11(4):466-75.

11. Viña-Alminia J, Peñarrocha-Diago M, Peñarrocha-Diago M. Influence of perforation of the sinus membrane on the survival rate of implants placed after direct sinus lift. Literature update. Med Oral Patol Oral Cir Bucal 2009; 14(3):E133-6.

12. Ardekian L, Oved-Peleg E, Mactei EE, Peled M. The clinical significance of sinus membrane perforation during augmentation of the maxillary sinus. J Oral Maxillofac Surg 2006; 64(2):277-82.

\section{Endereço para correspondência:}

Assis Filipe Medeiros Albuquerque Universidade Federal do Rio Grande do Norte (UFRN) Departamento de Odontologia

Área de Cirurgia e Traumatologia Buco-Maxilo-Facial Av. Senador Salgado Filho, 1787 - Bairro Lagoa Nova $5905600 \quad$ Natal/RN

Fones: 5585 99444019; 3246-2373

E-mail: assis_filipe@hotmail.com.br 